Pacific Journal of Mathematic 


\title{
CHARACTERIZATION OF THE CONTINUOUS IMAGES OF ALL PSEUDO-CIRCLES
}

\author{
LAWRENCE FEARNLEY
}

\begin{abstract}
The purpose of this paper is to establish a characterization of the continuous images of all pseudo-circles, and develop techniques which may be used in the further investigation of the mapping properties of pseudo-circles. The principal conclusions drawn in this paper from this characterization are the theorems that every planar circularly chainable continuum is the continuous image of a pseudo-circle and every snake-like continuum is the continuous image of a pseudo-circle.
\end{abstract}

The class of pseudo-circles may be defined to be the class of hereditarily indecomposable circularly chainable continua such that, if $M$ is a pseudo-circle, then $M$ is the intersection of the sets of points of a sequence $D_{1}, D_{2}, D_{3}, \cdots$ of circular chains having the properties that: (1) $D_{i+1}$ is crooked in $D_{i}, i=1,2,3, \cdots$, (2) $D_{i+1}$ has unit winding number in $D_{i}, i=1,2,3, \cdots$ and (3) the mesh of $D_{i}$ approaches zero as $i$ increases without bound. Thus, every nondegenerate proper subcontinuum of a pseudo-circle is a pseudo-arc and every pseudo-circle can be embedded in the plane. In view of the relationships between pseudo-arcs and pseudo-circles and the fact that pseudo-arcs are known to have important mapping properties, a number of questions have been raised in the literature regarding the mapping characteristics of pseudo-circles.

We now amplify the foregoing statements. In a recent paper [6] the author has established a global characterization of the continuous images of the pseudo-arc [1], [8], [11] which is similar in certain respects to the well known Hahn-Mazurkiewicz characterization of the continuous images of the arc. This result, which was also established independently by A. Lelek [9], constituted an answer to a question raised by R. H. Bing at the Summer Institute on Set Theoretic Topology, 1955 [3]. In addition, this characterization proved to be useful in [6] in showing that there does not exist any local topological property which characterizes the continuous images of the pseudo-arc. Furthermore, in a subsequent paper of this author [7] the characterization of the continuous images of the pseudo-arc was used to establish properties of topological operations on the class of continuous images of all snake-like continua.

The purpose of this present paper is to establish a corresponding characterization of the continuous images of all pseudo-circles [2, p. 48] This characterization will be expressed in a manner which is formally 
similar to that given for the continuous images of the pseudo-arc in [6]. However, the development of this result involves establishing preliminary theorems on cyclic orderings, winding numbers of circular $p$-chain refinements and the composition properties of crooked and noncrooked cyclic $r$-patterns. In addition a concept of linear representation of a cyclic $r$-pattern which is similar to the concept of universal covering space is introduced and used strongly in proving the principal preliminary theorem of this paper. Thus the development of this characterization of the continuous images of all pseudo-circles is substantially different from that of the characterization of the continuous images of the pseudo-arc. Among the further results presented in this paper is the theorem that every planar circularly chainable continuum is a continuous image of a pseudo-circle. This latter result is analogous to the corresponding theorem for the pseudo-arc obtained by J. Mioduszewski [10] and this author [6]. It is also proved that all snake-like continua are continuous images of pseudo-circles.

2. Preliminaries. The more standard terms used in this paper are defined in [12] or in the other appropriately indicated references. In addition, we shall define a number of special terms to be used throughout this paper. In general, these terms and notations were suggested by those used by Bing in [1] and [2] and those used by the author in [6].

It will be convenient in this development to use a modified form of the standard modular notation for cyclic systems. Specifically, the notation $k \bmod n$, where $k$ is a nonnegative integer and $n$ is a positive integer, will be used to denote the remainder obtained in dividing $k$ by $n$. Thus, for example, the relationship $n=0(\bmod n)$ will be written $n \bmod n=0$ in this paper to facilitate the presentation of results which involve more than one cyclic system.

Definition 1. A p-chain will be defined to be a finite sequence of sets each of which, except the last, intersects its successor in the sequence. A circular p-chain will be defined to be a $p$-chain in which the first and last links intersect. The members of the $p$-chain or circular $p$-chain will be called links and the notations $P=P(0, n)=$ $\left(p_{0}, p_{1}, \cdots, p_{n}\right)$ will be used to denote the $p$-chain or circular $p$-chain whose links are $p_{0}, p_{1}, \cdots, p_{n}$.

Definition 2. A function $f$ defined by a collection of ordered pairs of integers $((k, f(k)),(k+1, f(k+1)), \cdots,(k+n, f(k+n)))$ will be said to be an r-pattern if $|f(i)-f(j)| \leqq 1$ whenever $|i-j| \leqq 1$, $k \leqq i, j \leqq k+n$. If $g=((0, g(0)),(1, g(1)), \cdots,(n, g(n)))$ has range $(0,1, \cdots, m)$, for some positive integer $m$, and $|g(i)-g(j)| \bmod m \leqq 1$ 
whenever $|i-j| \bmod n \leqq 1,0 \leqq i, j \leqq n$, then $g$ will be said to be a cyclic r-pattern.

Definition 3. If $P=\left(p_{0}, p_{1}, \cdots, p_{n}\right)$ and $Q=\left(q_{0}, q_{1}, \cdots, q_{m}\right)$ are $p$-chains such that each link $p_{i}$ of $P$ is a subset of some link $q_{f(i)}$ of $Q$ and the sequence $f$ of ordered pairs $((0, f(0)),(1, f(1)), \cdots,(n, f(n)))$ is an $r$-pattern with range $(0,1, \cdots, m)$ then $f$ will be said to be an $r$-pattern of $P$ in $Q$. If $P$ and $Q$ are circular $p$-chains and $f$ is a cyclic $r$-pattern then $f$ will be said to be a cyclic r-pattern of $P$ in $Q$.

Definition 4. Two cyclic r-patterns

$$
f=((0, f(0)),(1, f(1)), \cdots,(n, f(n)))
$$

and

$$
g=((0, g(0)),(1, g(1)), \cdots,(n, g(n)))
$$

will be defined to be similar if $f$ and $g$ have the same set $(0,1, \cdots, m)$ as range and these is an integer $h$ such that one of the following two conditions holds:

(a) $f(i)=(h+g(i)) \bmod (m+1), 0 \leqq i \leqq n$,

(b) $f(i)=(h-g(i)) \bmod (m+1), 0 \leqq i \leqq n$.

Definition 5. Two cyclic $r$-patterns

$$
f=((0, f(0)),(1, f(1)), \cdots,(n, f(n)))
$$

and

$$
g=((0, g(0)),(1, g(1)), \cdots,(n, g(n)))
$$

will be defined to be equivalent if $f$ and $g$ have the same set $(0,1, \cdots, m)$ as range and there is an integer $h$ such that one of the following two conditions holds:

(a) $f(i)=g((h+i) \bmod (n+1)), 0 \leqq i \leqq n, \cdot$

(b) $f(i)=g((h-i) \bmod (n+1)), 0 \leqq i \leqq n$.

If $f$ and $g$ are cyclic $r$-patterns such that either $g$ is equivalent to a cyclic $r$-pattern similar to $f$ or $g$ is similar to a cyclic $r$-pattern equivalent to $f$, then $g$ will be referred to as an adjustment of $f$. We note that each of the relationships "similarity", "equivalence" and "adjustment" are equivalence relationships.

Definition 6. If $f=((0, f(0)),(1, f(1)), \cdots,(n, f(n)))$ is a cyclic $r$-pattern with range $(0,1, \cdots, m)$, then the sequence of ordered pairs of integers $((f(0), f(1)),(f(1), f(2)), \cdots,(f(n-1), f(n)),(f(n), f(0)))$ will be said to be the characteristic sequence of $f$ and will be denoted by 
$C(f)$. The occurences of the ordered pairs $(m, 0)$ and $(0, m)$ in $C(f)$ will be referred to as the positive transitions of $f$ and the negative transitions of $f$, respectively. The number of positive transitions of $f$ minus the number of negative transitions of $f$ will be said to be the winding number of $f$. In this development the cyclic r-patterns whose winding numbers have unit absolute value will be of particular importance. A cyclic $r$-pattern whose winding number has absolute value equal to 1 will be said to be a monocyclic r-pattern.

DEFINITION 7. If $f$ is a cyclic $r$-pattern such that $f(0)=0$, then $f$ will be said to have canonical form. Let $f$ be a cyclic $r$-pattern having canonical form and let $a$ and $b$ be elements of the domain of $f$ such that $0<a<b$ and such that no integer of the sequence $f(a), f(a+1), \cdots, f(b)$ is zero. Then the sequence of ordered pairs of integers $(a, f(a)),(a+1, f(a+1)), \cdots,(b, f(b))$ will be defined to be a primary r-pattern of $f$.

Definition 8. If $f=((0, f(0)),(1, f(1)), \cdots,(n, f(n)))$ is a cyclic $r$-pattern with range $(0,1, \cdots, m)$ and

$$
g=((k, g(k)),(k+1, g(k+1)), \cdots,(k+t, g(k+t)))
$$

is an $r$-pattern such that (1) $n \leqq t$ and $(2)|f(i)-g(j)| \bmod (m+1)=0$ whenever $i$ is the least nonnegative integer such that

$$
|i-j| \bmod (n+1)=0, k \leqq j \leqq k+t,
$$

then $g$ is defined to be a linear representation of $f$.

Definition 9. A p-chain $P$ will be said to be a refinement of a $p$-chain $Q$ if there is an $r$-pattern of $P$ in $Q$. If $P$ is a circular $p$-chain which has a cyclic $r$-pattern in a circular $p$-chain $Q$ then $P$ will be said to be a circular refinement of $Q$.

The following types of refinements and circular refinements will be distinguished:

Definition 10. A p-chain $P$ will be said to be a normal refinement of a $p$-chain $Q$ if there is an $r$-pattern

$$
f=((k, f(k),(k+1, f(k+1)), \cdots,(k+n, f(k+n)))
$$

with range $(h, h+1, \cdots, h+m)$ of $P$ in $Q$ such that $f(k)=h$ and $f(k+n)=h+m$. If a $p$-chain $P$ has an $r$-pattern $f$ in a $p$-chain $Q$ and each link $p_{i}$ of $P$ is the same set as the link $q_{f^{(i)}}$ of $Q$, then $P$ will be defined to be a principal refinement of $Q$. A $p$-chain $P$ will be said to be a crooked refinement of a $p$-chain $Q$ if there is an 
$r$-pattern $f$ of $P$ in $Q$ such that if $i$ and $j$ are integers of the domain of $f, i<j$ and $|f(i)-f(j)|>2$, then there are integers $u$ and $v$ with the properties that $i<u<v<j,|f(u)-f(j)| \leqq 1$ and $|f(v)-f(i)| \leqq 1$. If $f$ is an $r$-pattern having the properties described in the definition of "crooked refinement" we shall refer to $f$ as a crooked r-pattern.

DEFINITION 11. If $P$ is a circular $p$-chain having a cyclic $r$-pattern $f$ in a circular $p$-chain. $Q$, and each link $p_{i}$ of $P$ is the same set as the link $q_{f(i)}$ of $Q$, then $P$ will be said to be a principal circular refinement of $Q$. A circular $p$-chain $P$ will be said to be crooked in a circular $p$-chain $Q$ if there is a cyclic $r$-pattern $f$ of $P$ in $Q$ such that whenever $g$ is an adjustment of $f$ having canonical form and $r$ is a primary $r$-pattern of $g$, then $r$ is a crooked $r$-pattern. We shall refer to a cyclic $r$-pattern $f$ having the properties described in the definition of "crooked refinement" for circular p-chains as a crooked cyclic r-pattern.

In any type of refinement of a $p$-chain $P$ in a $p$-chain $Q$, there may be several $r$-patterns of $P$ in $Q$ having the appropriate properties. However, in referring to a refinement whose existence has been hypothesized or otherwise established, we will assume that a particular $r$-pattern has been chosen and that this $r$-pattern will remain fixed throughout the given argument. Thus, in these circumstances, we will speak of "the" $r$-pattern of a $p$-chain $P$ in a $p$-chain $Q$. A similar assumption will also be made with respect to circular refinements.

The concept of circular $p$-chainability of a continuum is now introduced.

Definition 12. A continuum $H$ will be said to be circularly $p$-chainable if there is a sequence $P_{1}, P_{2}, P_{3}, \ldots$ of circular $p$-chains such that for each positive integer $i$ :

(a) The union of the elements of $P_{i}$ is $H$.

(b) There is a monocyclic $r$-pattern $P_{i+1}$ in $P_{i}$.

(c) The diameter of each link of $P_{i}$ is less than $1 / i$.

(d) The closure of each link of $P_{i+1}$ is a subset of the link of $P_{i}$ to which it corresponds under the monocyclic $r$-pattern of $P_{i+1}$ in $P_{i}$.

A sequence of circular $p$-chains $P_{1}, P_{2}, P_{3}, \cdots$ having these properties with respect to the continuum $H$ will be said to be cyclically associated with $H$.

It will be shown that the property of circular $p$-chainability is a characterizing property of the class of continuous images of all pseudo-circles. This property will also be of fundamental importance in the development of the related results mentioned in the introductory section of this paper. 
3. Properties of cyclic $r$-patterns. In this section, we shall establish the principal combinatorial properties of cyclic $r$-patterns. These results will be used strongly in $\S 4$ in establishing the major theorems of this paper.

THEOREM 3.1. The absolute value of the winding number of a cyclic r-pattern is invariant under the operations of similarity and equivalence.

Proof. Let $f=((0, f(0)),(1, f(1)), \cdots,(n, f(n)))$ be a cyclic $r$ pattern with range $(0,1, \cdots, m)$ and winding number $w$. We first prove that if $g$ is a cyclic $r$-pattern similar to $f$, then $g$ has winding number $\pm w$. Two cases will be considered.

Case 1. There is an integer $k$ such that, for each integer $i$ in the domain of $f f(i)=(k+g(i)) \bmod (m+1)$. In this case we may assume that $k$ is a positive integer less than $m+1$. Then the positive transitions of $g$ are in one-to-one correspondence with the occurences of the ordered pair $(k-1, k)$ in the characteristic sequence $C(f)$ of $f$. The negative transitions of $g$ are in one-to-one correspondence with the occurences of $(k, k-1)$ in $C(f)$. We now proceed to obtain a relationship involving the number $u$ of occurences of $(k-1, k)$ in $C(f)$, the number $v$ of occurence of $(k, k-1)$ in $C(f)$ and the winding number $w$ of $f$.

To do this, let $i_{1}, i_{2}, \cdots, i_{t}$ be the maximal increasing sequence of integers of the domain of $f$ such that, for each number $i_{j}$ of this sequence, $\left(f\left(i_{j}\right), f\left(\left(i_{j}+1\right) \bmod (n+1)\right)\right)$ is either a positive transition of $f$ or a negative transition of $f$. Next observe that $u$ is equal to the number of occurences of $(k-1, k)$ in the collection

$$
\begin{array}{r}
\left(\left(f\left(i_{1}\right), f\left(i_{1}+1\right),\left(f\left(i_{1}+1\right), f\left(i_{1}+2\right)\right), \cdots,(f(n-1), f(n)),\right.\right. \\
\left.(f(n), f(0)),(f(0), f(1)), \cdots,\left(f\left(i_{1}-1\right), f\left(i_{1}\right)\right)\right) .
\end{array}
$$

Thus $u$ is equal to the sum of the integers $u_{1}, u_{2}, \cdots, u_{t}$ which are, respectively, the number of occurences of $(k-1, k)$ in the collections:

$$
\begin{aligned}
& C_{1}=\left(\left(f\left(i_{1}+1\right), f\left(i_{1}+2\right)\right),\left(f\left(i_{1}+2\right), f\left(i_{1}+3\right)\right), \cdots,\left(f\left(i_{2}-1\right), f\left(i_{2}\right)\right)\right), \\
& C_{2}=\left(\left(f\left(i_{2}+1\right), f\left(i_{2}+2\right)\right),\left(f\left(i_{2}+2\right), f\left(i_{2}+3\right)\right), \cdots,\left(f\left(i_{3}-1\right), f\left(i_{3}\right)\right)\right), \\
& \vdots \\
& C_{t}=\left(\left(f\left(i_{t}+1\right), f\left(i_{t}+2\right)\right),\left(f\left(i_{t}+2\right), f\left(i_{t}+3\right)\right), \cdots,(f(n-1), f(n)),\right. \\
& \left.\quad(f(n), f(0)),(f(0), f(1)), \cdots,\left(f\left(i_{1}-1\right), f\left(i_{1}\right)\right)\right) .
\end{aligned}
$$

In a similar manner, $v$ can be expressed as the sum of the numbers $v_{1}, v_{2}, \cdots, v_{t}$ of occurences of $(k, k-1)$ in the collections 
$C_{1}, C_{2}, \cdots, C_{t}$, respectively. Now, for each integer $j, 1 \leqq j \leqq t$, the two elements of a given ordered pair of the collection $C_{j}$ differ by at most 1 , the second member of each ordered pair of $C_{j}$ is equal to the first member of the succeeding ordered pair of $C_{j}$, if any. Furthermore, if $\left(f\left(i_{j}\right), f\left(i_{j}+1\right)\right)$ and $\left(f\left(i_{j+1}\right), f\left(i_{j+1}+1\right)\right)$ are both positive transitions of $f$, then $f\left(i_{j}+1\right)=0$ and $f\left(i_{j+1}\right)=m$ so that $u_{j}-v_{j}=1$. Similarly, if $\left(\left(f\left(i_{j}\right), f\left(i_{j}+1\right)\right)\right.$ and $\left(f\left(i_{j+1}\right), f\left(i_{j+1}+1\right)\right)$ are both negative transitions of $f$, then $u_{j}-v_{j}=-1$. In both of the remaining situations we obtain the result $u_{j}-v_{j}=0$. Thus, we conclude that the winding number $w$ of $f$ is equal to the winding number $u-v$ of $g$ and the proof for this case is complete.

Case 2. There is an integer $k$ such that, for each integer $i$ in the domain of $f, f(i)=(k-g(i)) \bmod (m+1)$. In this case we may assume that $m \leqq k \leqq 2 m+1$. Furthermore, if $k=m$ or $k=2 m+1$, then the occurences of the positive transitions and negative transitions of $f$ are in a one-to-one correspondence with the negative transitions and positive transitions, respectively, of $g$. In this situation, the winding number of $g$ is opposite in sign but equal in absolute value to the winding number of $f$. If $m<k<2 m+1$, the positive transitions of $g$ are in one-to-one correspondence with the occurences of $(k+1, k)$ in $C(f)$, and the negative transitions of $g$ are in one-to-one correspondence with the occurences of $(k, k+1)$ in $C(f)$. These situations, in reverse order, were considered in Case 1 and we conclude, in this case, that the winding number of $g$ is equal in absolute value, although opposite in sign, to the winding number of $f$.

Finally, we consider the relationships of the winding numbers of equivalent cyclic $r$-patterns $f$ and $g$. In the case that $f$ and $g$ satisfy condition (a) of Definition 5, the sequence of integers $g(0), g(1), \cdots, g(n)$ is a cyclic permutation of the sequence of integers $f(0), f(1), \cdots, f(n)$. If $f$ and $g$ satisfy condition (b) of Definition 5 , the sequence $g(0), g(1), \cdots$, $g(n)$ is a cyclic permutation of the sequence $f(n), f(n-1), \cdots, f(0)$. If follows, in both cases, that the absolute value of the winding number of $f$ is equal to the absolute value of the winding number of $g$.

Therefore, the absolute value of the winding number of a cyclic $r$-pattern is invariant under the operations of similarity and equivalence.

COROLlaRY. If $f$ is a cyclic r-pattern with range $(0,1, \cdots, m)$ and $k$ is an integer, $0 \leqq k \leqq m$, then the integer obtained by subtracting the number of occurences of $((k+1) \bmod (m+1), k)$ in $C(f)$ from the number of occurences of $(k,(k+1) \bmod (m+1))$ in $C(f)$ is equal to the winding number of $f$.

THEOREM 3.2. If $f$ and $g$ are cyclic $r$-patterns such that $f g$ is a defined composite function, then fg is a cyclic r-pattern. Furthermore, 
the winding number of $f g$ is equal to the product of the winding numbers of $f$ and $g$.

Proof. Let $f=((0, f(0)),(1, f(1)), \cdots,(n, f(n)))$ be a cyclic $r$ pattern with range $(0,1, \cdots, m)$ and let

$$
g=((0, g(0)),(1, g(1)), \cdots,(t, g(t)))
$$

be a cyclic $r$-pattern with range $(0,1, \cdots, n)$. Then, since $f$ is a cyclic $r$-pattern, $|f g(i)-f g(j)| \bmod m \leqq 1$ whenever

$$
|g(i)-g(j)| \bmod n \leqq 1,0 \leqq g(i), g(j) \leqq n .
$$

Furthermore, since $g$ is a cyclic $r$-pattern, $|i-j| \bmod t \leqq 1$ implies that

$$
|g(i)-g(j)| \bmod n \leqq 1,0 \leqq i, j \leqq t .
$$

Thus $|f g(i)-f g(j)| \bmod m \leqq 1$ whenever

$$
|i-j| \bmod t \leqq 1,0 \leqq i, j \leqq t,
$$

so that $f g$ is a cyclic $r$-pattern.

Now, let $h$ and $k$ be the winding numbers of $f$ and $g$, respectively, and let $i$ be an integer such that an occurence of

$$
(f g(i), f g((i+1) \bmod (t+1)))
$$

is either a positive or negative transition of $f g$. Then $g(i)$ and $g((i+1) \bmod (t+1))$ are distinct integers such that

$$
|g(i)-g((i+1) \bmod (t+1))| \bmod n \leqq 1 .
$$

In general, if an occurence of an ordered pair of integers $(a, b)$ in $C(f g)$ is a transition of $f g$, there is an integer $u$ in the domain of $f$, and hence in the range of $g$, such that either $f(u)=\alpha$ and $f((u+1) \bmod (n+1))=b$ or $f(u)=b$ and $f((u+1) \bmod (n+1))=a$. From the Corollary to Theorem 3.1, the number of occurences of $(u,(u+1) \bmod (n+1))$ in $C(g)$ minus the number of occurences of $((u+1) \bmod (n+1), u)$ in $C(g)$ is equal to the winding number $k$ of $q$. In addition, the number of occurences of $(a, b)$ in $C(f)$ minus the number of occurences of $(b, a)$ in $C(f)$ is equal to either $h$ or $-h$ according as an occurence of $(a, b)$ in $C(f)$ is a positive or negative transition of $f$. Therefore, the winding number of $f g$ is equal to the product $h k$.

We note that the class of monocyclic $r$-patterns is closed under the operation of function composition. The theorem which follows is the principal theorem involving the combinatorial and refinemental relationships among planar circular $p$-chains. In particular, it is an important preliminary theorem to the major theorems of this paper. 
THEOREM 3.3. If $f$ and $g$ are monocyclic r-patterns having identical ranges, then there exist monocyclic r-patterns $r$ and $s$ such that $f r$ and gs are defined composite functions and $f r=g s$.

The proof of this theorem is somewhat involved and it will be convenient to first establish the following two lemmas.

LEMMA 3.3.1. Let

$$
f=((0, f(0)),(1, f(1)), \cdots,(h, f(h)))
$$

and

$$
g=((0, g(0)),(1, g(1)), \cdots,(k, g(k)))
$$

be monocyclic $r$-patterns having identical ranges, let $f_{1}$ and $g_{1}$ be linear representations of $f$ and $g$, respectively, and let $r_{1}$ and $s_{1}$ be $r$-patterns having the same set $(0,1, \cdots, n)$ as domain and satisfying the following conditions.

(1) $f_{1} r_{1}$ and $g_{1} s_{1}$ are defined composite functions and $f_{1} r_{1}=g_{1} s_{1}$

(2) $\left|r_{1}(0)-r_{1}(n)\right|=h+1$.

(3) $\left|s_{1}(0)-s_{1}(n)\right|=k+1$.

Then there are monocyclic r-patterns $r$ and $s$ such that fr and $g s$ are defined and $f r=g s$.

Proof. We shall define $r$ and $s$ in a parallel manner. First, $r$ is defined to have the same domain $(0,1, \cdots, n)$ as $r_{1}$ and, for each integer $i$ in the domain of $r, r(i)$ is defined to be the least nonnegative integer such that $\left|r(i)-r_{1}(i)\right| \bmod (h+1)=0$. The function $s$ is then defined by replacing the symbols $r, r_{1}$ and $h$ in the preceding definition by $s, s_{1}$ and $k$, respectively.

Now, the range of $r_{1}$ is identical with the domain of $f_{1}$ and $f_{1}$ is a linear representation of $f$, so that the range of $r_{1}$ includes integers of the form $a, a+1, \cdots, a+h$. Thus, it follows that the range of $r$ is identical with the domain of $f$. Similarly, the domain of $g$ is identical with the range of $s$. Hence $f r$ and $g s$ are defined composite functions.

To see that $r$ is a monocyclic $r$-pattern, we note first, since $r_{1}$ is an $r$-pattern, that if $i$ and $j$ are integers such that $0 \leqq i, j \leqq n$, and $|i-j| \leqq 1$, then $\left|r_{1}(i)-r_{1}(j)\right| \leqq 1$. Hence $|r(i)-r(j)| \bmod h \leqq 1$. If $|i-j|=n$ it follows from condition (2) of the hypothesis that $\left|r_{1}(i)-r_{1}(j)\right| \bmod (h+1)=0$ so that in this case also $|r(i)-r(j)| \bmod h \leqq 1$. To complete the proof that $r$ is a monocyclic $r$-pattern we need to show that the absolute value of the winding number of $r$ is equal to 1. We do this by observing that $r_{1}$ is a linear representation of $r$ and that $\left|r_{1}(0)-r_{1}(n)\right|=h+1$. Thus the absolute value of the winding 
number of $r$ is equal to 1 . Therefore $r$ is a monocyclic $r$-pattern. A similar argument shows that $s$ is a monocyclic $r$-pattern.

Finally, let $i$ be an integer in the common domain of $r$ and $s$. We shall show that $f r(i)=g s(i)$. From the definition of $r, r(i)$ is the least nonnegative integer such that $\left|r(i)-r_{1}(i)\right| \bmod (h+1)=0$. Thus, if $(0,1, \cdots, m)$ is the common range of $f$ and $g$, it follows from the definition of "linear representation" that

$$
\left|f r(i)-f_{1} r_{1}(i)\right| \bmod (m+1)=0 .
$$

Furthermore, in a similar manner $\left|g s(i)-g_{1} s_{1}(i)\right| \bmod (m+1)=0$. In addition, from condition (1) of the hypothesis of the lemma, $f_{1} r_{1}(i)=g_{1} s_{1}(i)$ and $0 \leqq f r(i), g s(i) \leqq m$. Therefore we conclude that $f r(i)=g s(i)$ and the proof is complete.

LEMMA 3.3.2. Let $f$ and $g$ be monocyclic r-patterns having identical ranges, let $f_{1}$ and $g_{1}$ be monocyclic $r$-patterns equivalent to $f$ and $g$, respectively, and suppose $r_{1}$ and $s_{1}$ are monocyclic $r$-patterns such that $f_{1} r_{1}$ and $g_{1} s_{1}$ are defined composite functions and $f_{1} r_{1}=g_{1} s_{1}$. Then, there exist monocyclic $r$-patterns $r$ and $s$ such that fr and $g s$ are defined composite functions and $f r=g s$.

Proof. Let $f=((0, f(0)),(1, f(1)), \cdots,(h, f(h)))$, let $g=((0, g(0))$, $(1, g(1)), \cdots,(k, g(k)))$ and let $(0,1, \cdots, m)$ be the common range of $f$ and $g$. Then, since $f_{1}$ is equivalent to $f$, there exists an integer $v_{s}$ such that one of the following two conditions is satisfied:

(1) $f_{1}(i)=f((u+i) \bmod (h+1)), 0 \leqq i \leqq h$,

(2) $f_{1}(i)=f((u-i) \bmod (h+1)), 0 \leqq i \leqq h$.

Similarly, since $g_{1}$ is equivalent to $g$, there exists an integer $v$ sucn that one of the following two conditions is satisfied.

(3) $g_{1}(i)=g((v+i) \bmod (h+1)), 0 \leqq i \leqq k$,

(4) $g_{1}(i)=g((v-i) \bmod (h+1)), 0 \leqq i \leqq k$.

We shall assume that $f_{1}$ satisfies condition (1) and that $g_{1}$ satisfies condition (3) since it will be seen that a similar proof may be followed in the remaining three cases. In this case we may also assume that $u$ is an integer such that $0 \leqq u \leqq h$ and $v$ is an integer such that $0 \leqq v \leqq k$.

Now, let $r_{1}=\left(\left(0, r_{1}(0)\right),\left(1, r_{1}(1)\right), \cdots,\left(n, r_{1}(n)\right)\right)$ and let

$$
s_{1}=\left(\left(0, s_{1}(0)\right),\left(1, s_{1}(1)\right), \cdots,\left(n, s_{1}(n)\right)\right) .
$$

We define $r$ and $s$ by the following two relationships:

(5) $\quad r(i)=\left(u+r_{1}(i)\right) \bmod (h+1), 0 \leqq i \leqq n$, and

(6) $s(i)=\left(v+s_{1}(i)\right) \bmod (k+1), 0 \leqq i \leqq n$.

Then the domain of $f$ and the range of $r$ are identical, and the 
domain of $g$ and the range of $s$ are identical. Thus $f r$ and $g s$ are defined composite functions. Furthermore, by Theorem 3.1, since $r$ and $r_{1}$ are similar and $s$ and $s_{1}$ are similar, it follows that $r$ and $s$ are monocyclic $r$-patterns. Finally, if $i$ is an integer of the set $(0,1, \cdots, n)$, then from (1) and (5),

$$
f r(i)=f\left(\left(u+r_{1}(i)\right) \bmod (h+1)\right)=f_{1} r_{1}(i) .
$$

Similarly, from (3) and (6),

$$
g s(i)=f\left(\left(v+s_{1}(i)\right) \bmod (k+1)\right)=g_{1} s_{1}(i) .
$$

Therefore, since $f_{1} r_{1}=g_{1} s_{1}$, we conclude that $r$ and $s$ are monocyclic $r$-patterns such that $f r=g s$.

Proof of Theorem 3.3. First note that for each monocyclic $r$-pattern there is an equivalent monocyclic $r$-pattern having positive winding number. Thus, by Lemma 3.3.2, we may assume without loss in generality that each of the cyclic $r$-patterns $f$ and $g$ have winding number equal to 1 . Now, let $f=((0, f(0)),(1, f(1)), \cdots,(h, f(h)))$, let $g=((0, g(0)),(1, g(1)), \cdots,(k, g(k)))$ and let $(0,1, \cdots, m)$ be the common range of $f$ and $g$. We shall establish the existence of the monocyclic $r$-patterns $r$ and $s$ by constructing two finite sequences of $r$-patterns related to $f$ and $g$, respectively, and showing that the last members of these sequences determine monocyclic $r$-patterns of the desired type.

We define $r$-patterns $f_{1}$ and $g_{1}$ in the following manner. Let $f_{1}$ be the $r$-pattern having domain $(0,1, \cdots, h+1)$ such that $f_{1}(0)=f(0)$ and, if $f_{1}(i)$ has been chosen, $0 \leqq i \leqq h$, then $f_{1}(i+1)$ is defined to be:

$$
\begin{array}{ll}
f_{1}(i)+1 & \text { if } f((i+1) \bmod (h+1))=(f(i)+1) \bmod (m+1), \\
f_{1}(i)-1 & \text { if } f((i+1) \bmod (h+1))=(f(i)+1) \bmod (m+1),
\end{array}
$$

or

$$
f_{1}(i) \quad \text { if } f((i+1) \bmod (h+1))=f(i) .
$$

The pattern $g_{1}$ is defined by replacing the symbols $f, f_{1}$ and $h$, in the preceding definition by $g, g_{1}$ and $k$, respectively. Then $f_{1}$ and $g_{1}$ are $r$-patterns which are linear representations of $f$ and $g$, respectively, and $f_{1}(h+1)-f_{1}(0)=g_{1}(k+1)-g_{1}(0)=m+1$.

Next we define $r$-patterns $f_{2}$ and $g_{2}$. Since we shall require, again, that the definitions have parallel form, it will be sufficient to define $f_{2}$. To do this, let $u$ be an integer such that $f_{1}(u)$ is a minimal element of the range of $f_{1}$. We define $f_{2}$ to be the $r$-pattern having the same domain as $f_{1}$ such that $f_{2}(0)=f_{1}(u)$ and, if $f_{2}(i)$ has been chosen, $0 \leqq i \leqq h$, then we define $f_{2}(i+1)$ to be equal to $f_{1}(i+u+1)$ for $0 \leqq i \leqq h-u$ and to be equal to $f_{1}(i+v-h)+(m+1)$ for 
$h-u<i \leqq h$. It follows that $f_{2}$ and $g_{2}$ are $r$-patterns which are linear representations of monocyclic $r$-patterns equivalent to $f_{1}$ and $g_{1}$, respectively.

We now define $r$-patterns $f_{3}$ and $g_{3}$ which are modifications of $f_{2}$ and $g_{2}$, respectively, such that $f_{3}(0)=g_{3}(0)$. Specifically, observe that one of the $r$-patterns $f_{2}$ and $g_{2}$ has a member of its range which is equal to the least member of the range of the other $r$-pattern. Without loss in generality we may assume that $f_{2}(0)$ is equal to some element of the range of $g_{2}$. Let $c$ be the greatest integer such that $f_{2}(0)=g_{2}(c)$, and let $g_{3}$ be defined by the relationships

$$
g_{3}(i)=g_{2}(c+i) \quad \text { for } \quad 0 \leqq i \leqq k-c,
$$

and

$$
g_{3}(i)=g_{2}(c+i-k-1)+(m+1) \quad \text { for } \quad k-c<i \leqq k+1 .
$$

We then define $f_{3}$ to be identical with $f_{2}$. Now, since $g_{2}$ is an $r$ pattern such that $g_{2}(k+1)-g_{2}(0)=m+1, g_{2}(0)$ is a minimal element of the range of $g_{2}$ and $c$ is the greatest integer such that $f_{2}(0)=g_{2}(c)$, it follows that $g_{3}(0)$ is a minimal element of the range of $g_{3}$. Furthermore, since the definition of $g_{3}$ in terms of $g_{2}$ is analogous to the definition of $g_{2}$ in terms of $g_{1}, g_{3}$ is a linear representation of some monocyclic $r$-pattern equivalent to $g$. Thus, the $r$-patterns $f_{3}$ and $g_{3}$ have the following properties: (1) $f_{3}(0)=g_{3}(0)$, (2) $f_{3}(0)$ and $g_{3}(0)$ are minimal elements of the ranges of $f_{3}$ and $g_{3}$, respectively, (3) $f_{3}$ is a linear representation of a monocyclic $r$-pattern equivalent to $f$, (4) $g_{3}$ is a linear representation of a monocyclic $r$-pattern equivalent to $g$ and $(5) f_{3}(h+1)=g_{3}(k+1)$.

Finally, we define $r$-patterns $f_{4}$ and $g_{4}$. To do this, note from properties (1), (2), (3) and (4) that either the range of $f_{3}$ is a subset of the range of $g_{3}$, or the range of $g_{3}$ is a subset of the range of $f_{3}$. Without loss in generality we may suppose that the range of $f_{3}$ is a subset of the range of $g_{3}$. Now, let $v$ be an integer such that $g_{3}(v)$ is a maximal element of the range of $g_{3}$. Then, from property (3) and the fact that $f_{3}(h+1)-f_{3}(0)=m+1$, the following expression defines a linear representation of a monocyclic $r$-pattern equivalent to $f$ :

$$
\begin{aligned}
& \left(\left(0, f_{3}(0)\right),\left(1, f_{3}(1)\right), \cdots,\left(h, f_{3}(h)\right),\left(h+1, f_{3}(0)+(m+1)\right),\right. \\
& \left(h+2, f_{3}(1)+(m+1)\right), \cdots,\left(2 h+1, f_{3}(h)+(m+1)\right), \\
& \left(2 h+2, f_{3}(0)+2(m+1),\left(2 h+3, f_{3}(1)+2(m+1)\right), \cdots,\right. \\
& \left.\left(w_{1}, f_{3}\left(b_{w_{1}}\right)+d_{w_{1}}(m+1)\right)\right),
\end{aligned}
$$

where $0 \leqq b_{w_{1}} \leqq h, 0 \leqq d_{w_{1}}$ and $w_{1}$ is the least integer such that $f_{3}\left(b_{w_{1}}\right)+d_{w_{1}}(m+1)=g_{3}(v)$. We define $f_{4}$ to be the $r$-pattern determined by the foregoing conditions. The $r$-pattern $g_{4}$ is defined in a similar 
although not precisely parallel manner. Specifically, we define $g_{4}$ to be the linear representation of a monocyclic $r$-pattern equivalent to $g$ determined by the following expression:

$$
\begin{aligned}
& \left(\left(0, g_{3}(0)\right),\left(1, g_{3}(1)\right), \cdots,\left(k, g_{3}(k)\right),\left(k+1, g_{3}(0)+(m+1)\right),\right. \\
& \left.\left(k+2, g_{3}(1)+(m+1)\right), \cdots,\left(w_{2}, g_{3}\left(b_{w_{2}}\right)+(m+1)\right)\right),
\end{aligned}
$$

where $0 \leqq b_{w_{2}} \leqq k$, and $w_{2}$ is the least integer greater than or equal to $k+1$ such that $g_{3}\left(b_{w_{2}}\right)+(m+1)=g_{3}(v)$.

It will now be shown that the $r$-patterns $f_{4}$ and $g_{4}$ determine monocyclic $r$-patterns $r$ and $s$ of the desired type. We shall use Lemmas 3.3.1 and 3.3.2 and certain properties of $p$-chains. Let $Q=$ $Q\left(0, f_{4}\left(w_{1}\right)\right)$ be a $p$-chain, let $P=P\left(0, w_{1}\right)$ be a $p$-chain which is a normal refinement of $Q$ having $r$-pattern $f_{4}$ in $Q$ and let $T=T\left(0, w_{2}\right)$ be a $p$-chain which is a principal normal refinement of $Q$ having $r$-pattern $g_{4}$ in $Q$. Then, by Theorem 3.2 of [6], there is a $p$-chain $S_{1}=S_{1}\left(0, z_{1}\right)$ such that $S_{1}$ is a normal refinement of $P$ and a principal normal refinement of $T$. By property (2) of $f_{3}$ and the definition of $f_{4}$, the sub-p-chain $P\left(w_{1}, h+1\right)$ of $P\left(w_{1}, 0\right)$ is a normal refinement of $Q\left(f_{4}\left(w_{1}\right), f_{4}(0)+(m+1)\right)$. Similarly, the sub-p-chain $T\left(w_{2}, k+1\right)$ of $T\left(w_{2}, 0\right)$ is a principal normal refinement of $Q\left(f_{4}\left(w_{1}\right), f_{4}(0)+(m+1)\right)$. Hence there is a sub-p-chain $S_{2}=S_{1}\left(z_{1}, z_{2}\right)$ of $S_{1}\left(z_{1}, 0\right)$ such that $S_{2}$ is a normal refinement of $P\left(w_{1}, h+1\right)$ and a principal normal refinement of $T\left(w_{2}, k+1\right)$. It follows that the $p$-chain sum $S=S(0, z)=S_{1}+S_{2}$ is a refinement of $P$ and a principal normal refinement of $T$ such that the last link of $S$ corresponds to the $(h+1)$-st link of $P$ under the $r$-pattern of $S$ in $P$, and the last like of $S$ corresponds to the $(k+1)$-st link of $T$ under the $r$-pattern of $S$ in $T$.

Now, by Lemma 3.3.2, we may assume without loss in generality that $f_{4}$ and $g_{4}$ are linear representations of $f$ and $g$, respectively. Next, we note that the condition that a refinement be a principal refinement in the proof of Theorem 3.2 of [6] and in the preceding paragraph is not essential and that equivalent results may be obtained by considering the corresponding $r$-patterns. In particular it may be concluded that if $P$ and $T$ are normal refinements of $Q$ such that each link of a refining $p$-chain is contained in exactly one link of the refined $p$-chain, then there is a $p$-chain $S$ which is a normal refinement of both $P$ and $T$ and is such that each link of the refining $p$-chain is contained in exactly one link of a refined $p$-chain. Thus, in this theorem, if $r_{1}$ is the $r$-pattern of $S$ in $P$ and $s_{1}$ is the $r$-pattern of $S$ in $T$, it may be assumed that $f_{4}, g_{4}, r_{1}$ and $s_{1}$ have been chosen so that $f_{4} r_{1}=g_{4} s_{1}$. Finally, $r_{1}(z)-r_{1}(0)=m+1$ and $s_{1}(z)-s_{1}(0)=m+1$. Therefore, by Lemma 3.3.1, there exist monocyclic $r$-patterns $r$ and $s$ such that $f r=g s$. 
REMARK. The foregoing theorem cannot be modified to state that if $f$ and $g$ are arbitrary cyclic $r$-patterns having identical ranges then there are cyclic $r$-patterns $r$ and $s$ such that $f r=g s$. The following example shows that in the case of cyclic $r$-patterns $f$ and $g$ having: winding numbers each equal to zero, such a theorem would be false.

Let

$$
f=((0,0),(1,1),(2,2),(3,1),(4,0)),
$$

and

$$
g=((0,1),(1,2),(2,0),(3,2),(4,1)) .
$$

If $s$ is a cyclic $r$-pattern $((0, s(0)),(1, s(1)), \cdots,(n, s(n)))$ whose range is identical with the domain of $g$, there are integers $h$ and $k$ in the domain of $s$ such that $|h-k| \bmod (n-1)=1, s(h)=2$ and $s(k)=1$ or 3. Thus $g s(h)=0$ and $g s(k)=2$. Now, suppose that there exists a cyclic $r$-pattern $r=((0, r(0)),(1, r(1)), \cdots,(n, r(n)))$ such that $f r=g s$. Then $|r(h)-r(k)| \bmod 4 \leqq 1, f r(h)=0$ and $f r(k)=2$, which is contrary to the definition of $f$.

We now consider the final theorem of this section.

THEOREM 3.4. Let $f$ be a monocyclic $r$-pattern and let $g$ be a crooked monocyclic r-pattern such that $f g$ is a defined composite function. Then $f g$ is a crooked monocyclic r-pattern.

Proof. First we show that if $f_{1}$ is an $r$-pattern and $g_{1}$ is a crooked $r$-pattern such that $f_{1} g_{1}$ is a defined composite function then $f_{1} g_{1}$ is a crooked $r$-pattern. Since $g_{1}$ is an $r$-pattern $\left|g_{1}(i)-g_{1}(j)\right| \leqq 1$ whenever $i$ and $j$ are integers of the domain of $g_{1}$ such that $|i-j| \leqq 1$. Furthermore, $\left|g_{1}(i)-g_{1}(j)\right| \leqq 1$ implies that $\left|f_{1} g_{1}(i)-f_{1} g_{1}(j)\right| \leqq 1$, since $f_{1}$ is an $r$-pattern. Thus $\left|f_{1} g_{1}(i)-f_{1} g_{1}(j)\right| \leqq 1$ whenever $i$ and $j$ are integers of the domain of $f_{1} g_{1}$ such that $|i-j| \leqq 1$. Hence $f_{1} g_{1}$ is an $r$-pattern. To show that $f_{1} g_{1}$ is a crooked $r$-pattern, let $i$ and $j$ be integers of the domain of $f_{1} g_{1}$ such that $i<j$ and $\left|f_{1} g_{1}(i)-f_{1} g_{1}(j)\right|>2$. Then $\left|g_{1}(i)-g_{1}(j)\right|>2$. Therefore, since $g_{1}$ is a crooked $r$-pattern, there are integers $u$ and $v$ such that $i<u<v<j,\left|g_{1}(u)-g_{1}(j)\right| \leqq 1$ and $\left|g_{1}(v)-g_{1}(i)\right| \leqq 1$. It follows that $\left|f_{1} g_{1}(u)-f_{1} g_{1}(j)\right| \leqq 1$ and $\left|f_{1} g_{1}(v)-f_{1} g_{1}(i)\right| \leqq 1, i<u<v<j$, so that $f_{1} g_{1}$ is a crooked $r$-pattern.

Now, let $f=((0, f(0)),(1, f(1)), \cdots,(n, f(n)))$ be a monocyclic $r-$ pattern with range $(0,1, \cdots, m)$, let $g=((0, g(0)),(1, g(1)), \cdots,(t, g(t)))$ be a crooked monocyclic $r$-pattern with range $(0,1, \cdots, n)$ and note, by Theorem 3.2 , that $f g$ is a monocyclic $r$-pattern. We choose $p$ to. be a monocyclic $r$-pattern such that $p$ is an adjustment of $f g$ and $p$ has canonical form. Then, since an adjustment is the composition of 
a similarity operation and an equivalence operation, there exist integers $h$ and $k$ such that

(1) $p(i)=(h+f g((k+i) \bmod (t+1))) \bmod (m+1), 0 \leqq i \leqq t$ or $p(i)$ is defined by an equation similar to (1) in which one or both of the first two plus signs are replaced by minus signs. Since a similar argument may be followed in each of these four cases, we shall assume that $0 \leqq h \leqq m, 0 \leqq k \leqq t$ and that $p(i)$ is determined by equation (1).

We now show that the cyclic $r$-pattern $p$ can be expressed as a composite function $f^{\prime} g^{\prime}$, where $f^{\prime}$ is an adjustment of $f$ having canonical form and $g^{\prime}$ is an adjustment of $g$ having canonical form. To do this, let $w$ be the integer such that

$$
0 \leqq w \leqq n \text { and }(w+g(k)) \bmod (n+1)=0 .
$$

Then, the cyclic $r$-patterns $f^{\prime}$ and $g^{\prime}$ are defined in the following manner:

(2) $f^{\prime}(i)=(h+f((n+1-w+i) \bmod (n+1))) \bmod (m+1)$,

$$
0 \leqq i \leqq n,
$$

(3) $g^{\prime}(i)=(w+g((k+i) \bmod (t+1))) \bmod (n+1), 0 \leqq i \leqq t$.

Thus from (1), (2) and (3) it follows that

(4) $p(i)=f^{\prime}\left(g^{\prime}(i)\right), 0 \leqq i \leqq t$.

Furthermore, by the choice of $w, g^{\prime}(0)=0$. In addition, from (4) and the fact that $p$ and $g^{\prime}$ are cyclic $r$-patterns having canonical form, it follows that $f^{\prime}(0)=0$ so that $f^{\prime}$ also has canonical form.

To complete the proof of this theorem, noting the result obtained in the first paragraph of the proof, it is sufficient to show that each primary $r$-pattern of $p$ is equivalent to the composite function of an arbitrary $r$-pattern and a crooked $r$-pattern. Let

$$
r=((u, p(u)),(u+1, p(u+1), \cdots,(v, p(v)))
$$

be a primary $r$-pattern of $p$. Then, neither the domain nor the range of $r$ contains the element zero. Hence, from (4) and the fact that $f^{\prime}$ and $g^{\prime}$ are cyclic $r$-patterns having canonical form, the sequence $g^{\prime}(u), g^{\prime}(u+1), \cdots, g^{\prime}(v)$ consists of integers each of which is nonzero. Thus, noting Definition 2, it follows that the sequence of ordered pairs of integers

( 5 ) $\quad r_{2}=\left(\left(u, g^{\prime}(u)\right),\left(u+1, g^{\prime}(u+1)\right), \cdots,\left(v, g^{\prime}(v)\right)\right)$

is a primary $r$-pattern of $g^{\prime}$. In a similar manner, if $a$ and $b$ are the minimum and maximum integers of the range of $r_{2}$, then

(6) $r_{1}=\left(\left(a, f^{\prime}(a)\right),\left(a+1, f^{\prime}(a+1), \cdots,\left(b, f^{\prime}(b)\right)\right)\right.$

is a primary $r$-pattern of $f^{\prime}$. Now, $g^{\prime}$ is a crooked cyclic $r$-pattern, so that $r_{2}$ is a crooked $r$-pattern. Furthermore, $r=r_{1} r_{2}$. Therefore, 
$r$ is a crokeed $r$-pattern and we conclude that $f g$ is a crooked monocyclic $r$-pattern.

4. Characterization of the continuous images of all pseudocircles. The purpose of this section is to establish the two principal theorems of this paper, that the class of continuous images of all pseudo-circles consists of exactly those continua which are circularly $p$-chainable, and that every planar circularly chainable continuum is a continuous image of a pseudo-circle.

In the presentation of the theorems of this section it will be convenient to omit the qualifying term "circular" in the expression "circular refinement" where no confusion is likely to result.

THEOREM 4.1. If a continuum $C$ is a continuous image of a pseudo-circle then there exists a sequence of circular p-chains cyclically associated with $C$ such that each refinement is determined by a monocyclic r-pattern having canonical form.

Proof. Let $M$ be a pseudo-circle and let $f$ be a continuous transformation with domain $M$ so that the continuous image of $M$ under $f$ is the continuum $C$. Then, from the definition of "pseudocircle" given in [2, p. 48], there is a sequence of planar circular chains $D_{1}, D_{2}, D_{3}, \cdots$ such that for each positive integer $i$ : (1) each link of $D_{i}$ is an open circular disk having diameter less than $1 / i,(2)$ the closure of each link of $D_{i+1}$ is a subset of some link of $D_{i}$, (3) each complementary domain of the union of the links $D_{i+1}$ contains a complementary domain of the union of the links of $D_{i}$, (4) if $E_{i}$ is a proper sub-chain of $D_{i}$ and $E_{i+1}$ is a sub-chain of $D_{i+1}$ contained in $E_{i}$, then $E_{i+1}$ is crooked in $E_{i}$, and (5) the intersection of the sets of points of the circular chains $D_{1}, D_{2}, D_{3}, \cdots$ is $M$.

From condition (1) the union of the links of each circular chain $D_{i}$ is topologically equivalent to the interior of an annular ring. Hence, from conditions (3) and (5), $M$ is a continuum which separates the plane into exactly two complementary domains having $M$ as their common boundary. It follows that the circularly chainable continuum $M$ is not a snake-like continuum. Now, noting condition (2), if $i$ is a positive integer, there is a cyclic $r$-pattern $g_{i}$ of $D_{i+1}$ in $D_{i}$ such that the closure of each link of $D_{i+1}$ is a subset of the link of $D_{i}$ to which it corresponds under $g_{i}$. Furthermore, it is easily seen that $g_{i}$ can be chosen so that $g_{i}(0)=0$. Therefore, since $D_{i}$ and $D_{i+1}$ are planar circular chains whose links are open disks and each complementary domain of $D_{i}$ is a subset of a complementary domain of $D_{i+1}$, it follows from the proof of Theorem 3 of [4] that the winding number of $g_{i}$ has unit absolute value. 
We denote the sequence of links of $D_{i}$ by $\left(d_{i c}, d_{i 1}, \cdots, d_{i n_{i}}\right)$ and indicate the circular $p$-chain $\left(f\left(d_{i 0} \cap M\right), f\left(d_{i 1} \cap M\right), \cdots, f\left(d_{i n_{i}} \cap M\right)\right)$ by $P_{i}$, for $i=1,2,3, \cdots$. Now, $M$ is a compact continuum, so that the function $f$ is uniformly continuous. Thus, a subsequence $P_{k_{1}}, P_{k_{2}}, P_{k_{3}}, \cdots$ of the sequence $P_{1}, P_{2}, P_{3}, \cdots$ may be chosen so that for each positive integer $i$, the diamenter of each link of $P_{k_{i}}$ is less than $1 / i$. In addition, noting Theorem 3.2, the composite function $g_{k_{i}} g_{k_{i}+1} \cdots g_{k_{i+1}-1}$ is a monocyclic $r$-pattern of $P_{k_{i+1}}$ in $P_{k_{i}}$ having canonical form, $i=1,2,3, \cdots$. In addition, the closure of each link of $P_{k_{i+1}}$ is a subset of the link of $P_{k_{i}}$ to which it corresponds under the cyclic $r$-pattern of $P_{k_{i+1}}$ in $P_{k_{i}}, i=1,2,3, \cdots$. Finally, from condition (5), above, if $i$ is a positive integer then the union of the links of $P_{k_{i}}$ is equal to the continuum $C$. Therefore, the sequence of circular $p$-chains satisfies each of the requirements of Definition 12 with respect to $C$ and each refinement is determined by a cyclic $r$-pattern having canonical form.

The theorem which follows constitutes the characterization of the continuous images of all pseudo-circles mentioned in the first paragraph of this section and discussed in the Introduction of this paper. In the proof of this theorem, strong use will be made of the combinatorial properties of cyclic $r$-patterns established in the preceding section.

THEOREM 4.2. In order that a continuum $C$ be a continuous image of a pseudo-circle it is necessary and sufficient that $C$ be circularly p-chainable.

Proof of necessity. This follows form Theorem 4.1.

Proof of sufficiency. First we show that if $P_{1}, P_{2}, P_{3}, \ldots$ is a sequence of circular $p$-chains which is cyclically associated with the continuum $C$, then there is a corresponding sequence of circular $p$ chains $T_{1}, T_{2}, T_{3}, \cdots$ which satisfies the requirements of Definition 12 with respect to $C$ and is also such that for each positive integer $i$ :

(1) $T_{i}$ is a principal refinement of $P_{i}$ having a monocyclic $r$-pattern in $P_{i}$.

(2) If $i>1$, then $T_{i}$ is a crooked refinement of $T_{i-1}$ having a crooked monocyclic $r$-pattern in $T_{i-1}$.

To do this, let $T_{1}$ be an arbitrary principal refinement of $P_{1}$ having a monocyclic $r$-pattern in $P_{1}$. Next, assume that for some positive integer $n, T_{i}$ has been defined for each positive integer $i$ less than or equal to $n$, and that $T_{i}$ satisfies the requirements of Definition 12 and conditions (1) and (2), above. Now consider the case that $i=n+1$.

Since the sequence $P_{1}, P_{2}, P_{3}, \cdots$ is cyclically associated with $C$ there is a monocyclic $r$-pattern $f_{n}$ of $P_{n+1}$ in $P_{n}$. In addition, from condition (1), above, for $i=n$, there is a monocyclic $r$-pattern $g_{n}$ of 
$T_{n}$ in $P_{n}$. Thus, $f_{n}$ and $g_{n}$ are monocyclic $r$-patterns whose ranges are identical. It follows, by Theorem 3.3, that there exist monocyclic $r$-patterns $r_{n+1}$ and $s_{n}$ such that the composite functions $f_{n} r_{n+1}$ and $g_{n} s_{n}$ are defined and $f_{n} r_{n+1}=g_{n} s_{n}$. We define a circular $p$-chain $Q_{n+1}$ to be the principal refinement of $P_{n+1}$ which has the monocyclic $r$-pattern $r_{n+1}$ in $P_{n+1}$ and show that then $Q_{n+1}$ is a refinement of $T_{n}$ having the monocyclic $r$-pattern $s_{n}$ in $T_{n}$. It is also shown that the closure of each link of $Q_{n+1}$ is a subset of the link of $T_{n}$ to which it corresponds under $s_{n}$. In order to establish these two assertions it is noted that $s_{n}$ is a cyclic $r$-pattern, the domain of $s_{n}$ is identical with the set of subscripts of the links of $Q_{n+1}$ and the range of $s_{n}$ is identical with the set of subscripts of the links of $T_{n}$. Hence, both of these assertions will be established if it is shown that the closure of each link of $Q_{n+1}$ is a subset of the link of $T_{n}$ to which it corresponds under $s_{n}$. Now, $Q_{n+1}$ is a principal refinement of $P_{n+1}$ having the monocyclic $r$-pattern $r_{n+1}$ in $P_{n+1}$, so that a link of $Q_{n+1}$ with subscript $i$ is the same set as the link of $P_{n+1}$ with subscript $r_{n+1}(i)$. In addition, from condition (d) of Definition 12 for the sequence $P_{1}, P_{2}, P_{3}, \cdots$, the closure of the link of $P_{n+1}$ with subscript $r_{n+1}(i)$ is a subset of the link of $P_{n}$ with subscript $f_{n} r_{n+1}(i)$. Thus, since $T_{n}$ is a principal refinement of $P_{n}$ having the monocyclic $r$-pattern $g_{n}$ in $P_{n}$, the closure of the link of $Q_{n+1}$ with subscript $i$ is a subset of any link of $T_{n}$ with subscript in the set of integers $g_{n}^{-1} f_{n} r_{n+1}(i)$. Therefore, since $f_{n} r_{n+1}=g_{n} s_{n}$, the closure of the link of $Q_{n+1}$ with subscript $i$ is a subset of the link of $T_{n}$ with subscript $s_{n}(i)$, as was to be shown.

We now define $T_{n+1}$ to be a principal refinement of $Q_{n+1}$ having a crooked monocyclic $r$-pattern in $Q_{n+1}$. It is easily seen that such a circular $p$-chain exists. In particular we can choose $T_{n+1}$ to be the principal refinement of $Q_{n+1}$ having the crooked monocyclic $r$-pattern in $Q_{n+1}$ described in the example of a pseudo-circle in [2, p. 48]. To see that $T_{n+1}$ satisfies conditions (1) and (2), above, let $c_{n+1}$ denote the crooked monocyclic $r$-pattern of $T_{n+1}$ in $Q_{n+1}$. Then, by Theorem 3.2, $r_{n+1} c_{n+1}$ is a monocyclic $r$-pattern. Furthermore, $T_{n+1}$ is a principal refinement of $P_{n+1}$ under the monocyclic $r$-pattern $r_{n+1} c_{n+1}$ of $T_{n+1}$ in $P_{n+1}$. In addition, from Theorem 3.2 and Theorem 3.4, $s_{n} c_{n+1}$ is a crooked monocyclic $r$-pattern of $T_{n+1}$ in $T_{n}$. Thus, the sequence of circular $p$-chains $T_{1}, T_{2}, T_{3}, \cdots$ satisfies the required conditions (1) and (2). It remains to show that the sequence $T_{1}, T_{2}, T_{3}, \cdots$ also satis fies the requirements of Definition 12 with respect to the conitnuum $C$. The requirements (a) and (c) of Definition 12 follow from property (1), above, and requirement (b) of Definition 12 follows from property (2). above. The final requirement (d) of Definition 12 is a consequence of the fact that, for each positive integer $i, T_{i+1}$ is a principal refinement of 
$Q_{i+1}$, together with the result established in the preceding paragraph that the closure of each link of $Q_{i+1}$ is a subset of the link of $T_{i}$ to which it corresponds under the cyclic $r$-pattern of $Q_{i+1}$ in $T_{i}$.

In this last section of the proof we construct a pseudo-circle $M$ and define a continuous transformation $f$ of $M$ onto $C$. To do this, let $T_{1}, T_{2}, T_{3}, \cdots$ be a sequence of circular $p$-chains cyclically associated with $C$ such that for each positive integer $i, T_{i+1}$ has a crooked monocyclic $r$-pattern in $T_{i}$. We construct a corresponding sequence of circular chains $D_{1}, D_{2}, D_{3}, \cdots$ in the plane in the following manner. Let $D_{1}$ be a circular chain having the same number of links as $T_{1}$ and such that the links of $D_{1}$ are open circular disks of diameters less than 1 whose union is contained in the plane. Next, suppose for each positive integer $i$ less than or equal to some positive integer $n$ that $D_{i}$ has been chosen and consider the case that $i=n+1$. Now, from the definition of $T_{n+1}$ as a principal refinement crooked in a circular $p$-chain $Q_{n+1}$, it may be seen that $T_{n+1}$ can be chosen to have any sufficiently large number of links. Thus, we may assume that $T_{n+1}$ has a sufficient number of links that there is a circular chain $D_{n+1}$ having the same cyclic $r$-pattern in $D_{n}$ as the cyclic $r$-pattern of $T_{n+1}$ in $T_{n}$. Since the winding number of $D_{n+1}$ in $D_{n}$ has unit absolute value, it follows from the proof of Theorem 4 of [4] that such a circular chain $D_{n+1}$ can be constructed in the plane. In addition we choose $D_{n+1}$ to be such that the links of $D_{n+1}$ are open circular disks of diameter less than $1 / n+1$ and the closure of each link of $D_{n+1}$ is a subset of the link of $D_{n}$ to which it corresponds under the cyclic $r$-pattern of $D_{n+1}$ in $D_{n}$. Then, the circular chains $D_{1}, D_{2}, D_{3}, \cdots$ have the properties required in the definition of "pseudo-circle" [2, p. 48] so that the intersection $M$ of the sets of points of $D_{1}, D_{2}, D_{3}, \cdots$ is a pseudo-circle.

We now define the continuous transformation $f$ of $M$ onto $C$. To facilitate the description of $f$ let $D_{i}$ be represented by the sequence of links $\left(d_{i 0}, d_{i 1}, \cdots, d_{i k_{i}}\right), i=1,2,3, \cdots$. Let $x$ be a point of $M$ and let the sequence of links $d_{1 u_{1}}$ of $D_{1}, d_{2 u_{2}}$ of $D_{2}, d_{3 u_{3}}$ of $D_{3}, \cdots$ be a sequence of open sets closing down on $x$ such that, for each positive integer $i, d_{i+1 u_{i+1}}$ corresponds to $d_{i u_{i}}$ under the cyclic $r$-pattern of $D_{i+1}$ in $D_{i}$. We define

$$
f(x)=\bigcap_{i=1}^{\infty} t_{i u_{i}}
$$

and note by conditions (c) and (d) of Definition 12 that $f(x)$ exists and is a single point. If a second sequence of links $d_{1 v_{1}}$ of $D_{1}, d_{2 v_{2}}$ of $D_{2}, d_{3 v_{3}}$ of $D_{3}, \cdots$ closes down on $x$ then, for each positive integer $i,\left|u_{i}-v_{i}\right| \bmod k_{i} \leqq 1$. Hence the links $t_{u_{i}}$ and $t_{v_{i}}$ of $T_{i}$ intersect, $i=1,2,3, \cdots$. It follows from condition (c) of Definition 12 that 


$$
t_{1 u_{1}} \cap t_{2 u_{2}} \cap t_{3 u_{3}} \cap \cdots=t_{1 v_{1}} \cap t_{2 v_{2}} \cap t_{3 v_{3}} \cap \cdots .
$$

Thus $f$ is a well defined transformation. To prove that $f$ is a continuous transformation of $M$ onto $C$, let $t$ be an open set in $C$ and let $m$ be an integer such that $t$ contains three consecutive links

$$
t_{m j}, t_{m(j+1) \bmod k_{m}}, t_{m\langle j+2) \bmod k_{m}} \text { of } T_{m} .
$$

Now, if $d_{1 u_{1}}, d_{2 u_{2}}, d_{3 u_{3}}, \cdots$ is a sequence of links closing down on a point of $d_{m(j+1) \mathrm{mcd}_{m}}$ then one of the links $d_{m j}, d_{m(j+1) \bmod k_{m}}$ and $d_{m(j+2) \text { mod } k_{m}}$ is

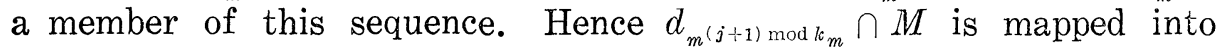
the subset $t_{m j} \cup t_{m(j+1) \bmod k_{m}} \cup t_{m(j+2) \bmod k_{m}}$ of $t$ under the transformation $f$. We conclude that $f$ is continuous and the compact set $f(M)$ is everywhere dense in $C$. This completes the proof.

We now present the second principal theorem of this paper. In the proof of this theorem, Theorem 4.3 , it is shown that all $p$-chainable continua [6] as well as all planar circularly chainable continua are circularly $p$-chainable. Thus, in particular, we obtain the additional result which is stated as Theorem 4.4. It is observed the Theorem 4.4 can alternatively be obtained from the statement of Theorem 4.3 rather than the proof of Theorem 4.3 in view of the result of Burgess [5, Th. 7] that every indecomposable chainable continuum is circularly chainable and the result of the author $[6$, Th. 4.1] that every chainable continuum is a continuous image of the pseudo-arc.

THEOREM 4.3. Every planar circularly chainable continuum is a continuous image of a pseudo-circle.

Proof. Let $C$ be a planar circularly chainable continuum. Then, from Theorem 9 of [4], $C$ is either a snake-like continuum or $C$ separates the plane into exactly two complementary domains having $C$ as their common boundary. We consider the two cases in turn.

Case 1. $C$ is a snake-like continuum. Then, by Theorems 3.5 and 4.1 of [6] there is a sequence of $p$-chains $P_{1}, P_{2}, P_{3}, \cdots$ such that for each positive integer $i$ :

(1) The union of the links of $P_{i}$ is $C$.

(2) $P_{i+1}$ is a normal refinement of $P_{i}$.

(3) The diameter of each link of $P_{i}$ is less than $1 / i$.

(4) The closure of each link of $P_{i+1}$ is a subset of the link of $P_{i}$ to which it corresponds under the $r$-pattern of $P_{i+1}$ in $P_{i}$. Now let $P_{i}$ be represented in the form $P_{i}\left(0, n_{i}\right), i=1,2,3, \cdots$ and consider the ordered $p$-chain sum

$$
Q_{i}\left(0, m_{i}\right)=P_{i}\left(0, n_{i}\right)+P_{i}\left(n_{i}, 0\right) .
$$


Since the first and last links of $Q_{i}\left(0, m_{i}\right)$ are identical, the $p$-chain $Q_{i}\left(0, m_{i}\right)$ is a circular $p$-chain. Furthermore, by property (1), above, the union of the links of $Q_{i}\left(0, m_{i}\right)$ is equal to $C$. Now, since $P_{i+1}$ is a normal refinement of $P_{i}$, it follows that the $r$-pattern of $P_{i+1}\left(0, n_{i+1}\right)$ in $P_{i}\left(0, n_{i}\right)$ together with the $r$-pattern of $P_{i+1}\left(n_{i+1}, 0\right)$ in $P_{i}\left(n_{i}, 0\right)$ determine a monocyclic $r$-pattern of $Q_{i+1}\left(0, m_{i+1}\right)$ in $Q_{i}\left(0, m_{i}\right)$. In addition, the closure of each link of $Q_{i+1}\left(0, m_{i+1}\right)$ is a subset of the link of $Q_{i}\left(0, m_{i}\right)$ to which it corresponds under the monocyclic $r$ pattern of $Q_{i+1}\left(0, m_{i+1}\right)$ in $Q_{i}\left(0, m_{i}\right)$. Thus, conditions (a), (b) and (d) of Definition 12 are satisfied by the sequence of circular $p$-chains

$$
Q_{1}\left(0, m_{1}\right), Q_{2}\left(0, m_{2}\right), Q_{3}\left(0, m_{3}\right), \cdots
$$

with respect to the continuum $C$. Finally, condition (c) of Definition 12 for the sequence

$$
Q_{1}\left(0, m_{1}\right), Q_{2}\left(0, m_{2}\right), Q_{3}\left(0, m_{3}\right), \cdots
$$

is a consequence of property (4), above, for the sequence $P_{1}, P_{2}, P_{3}, \cdots$. Therefore, $C$ is circularly $p$-chainable and we conclude, by Theorem 4.2 , that $C$ is a continuous image of a pseudo-circle.

Case 2. $C$ is a planar circularly chainable continuum which separates the plane into exactly two complementary domains. In this case we note by Theorem 7 of [4] that it may be assumed without loss in generality that for each positive number $\varepsilon, C$ can be irreducibly covered by a planar circular chain each of whose links is an open circular disk of diameter less than $\varepsilon$. Hence, there is a sequence of chains $D_{1}, D_{2}, D_{3}, \cdots$ in the plane such that for each positive integer $i$ :

(a) The union of the links $\left(d_{i 0}, d_{i 1}, \cdots, d_{i k_{i}}\right)$ of $D_{i}$ contains $C$.

(b) There is a cyclic $r$-pattern $f_{i}$ of $D_{i+1}$ in $D_{i}$.

(c) Each link of $D_{i}$ is an open circular disk of diameter less than $1 / i$.

(d) The closure of each link of $D_{i+1}$ is a subset of the link of $D_{i}$ to which it corresponds under $f_{i}$.

Then, since each link of each circular chain of the sequence $D_{1}, D_{2}$, $D_{3}, \cdots$ is a connected open set, it follows from the proof of Theorem 3 of [4] that for each positive integer $i$, the absolute value of the winding number of $f_{i}$ is equal to at most 1 . We show that for all but a finite number of values of $i, i=1,2,3, \cdots$, the absolute value of the winding number of $f_{i}$ is equal to 1 . For suppose that there is an increasing sequence of positive integers $h_{1}, h_{2}, h_{3}, \cdots$ such that for each positive integer $i, f_{h_{i}}$ has winding number zero. Then, by Theorem 3.1, $D_{h_{1}}, D_{h_{2}}, D_{h_{3}}, \cdots$ is a subsequence of $D_{1}, D_{2}, D_{3}, \cdots$ with the property that $D_{h_{i+1}}$ has a cyclic $r$-pattern with winding number 
zero in $D_{h_{i}}$ for $i=1,2,3, \cdots$. It follows that the sequence of open sets obtained by successively intersecting the links of $D_{h_{i+1}}$ with the set which is the union of the links of $D_{h_{i}}, i=1,2,3, \cdots$, is a linear chain. Thus we obtain the conclusion that $C$ is a snake-like continuum, which contradicts the fact that $C$ separates the plane. In view of the foregoing contradiction we conclude that there is a positive integer $n$ such that for each positive integer $j, f_{n+j}$ is a monocyclic $r$-pattern. Now let $D_{n+j} \cap C$ denote the circular $p$-chain

$$
\left(d_{n+j 0} \cap C, d_{n+j 1} \cap, C \cdots, d_{n+j k_{n+j}} \cap C\right), j=1,2,3, \cdots .
$$

Then the sequence of circular $p$-chains $D_{n+1} \cap C, D_{n+2} \cap C, D_{n+3} \cap C, \cdots$ satisfies each of the conditions of Definition 12 with respect to the continuum $C$. Therefore, by Theorem $4.2, C$ is a continuous image of a pseudo-circle.

THEOREM 4.4. Every snake-like continuum is a continuous image of a pseudo-circle.

Proof. This is a consequence of the argument given in Case 1 of the proof of Theorem 4.3.

The referee has noted that Theorem 3.3 together with the remark following this theorem suggest the question of whether or not there exists a result similar to Theorem 3.3 in the case that the winding numbers of the cyclic $r$-patterns are arbitrary positive integers. He has also suggested that it be mentioned that it is not known whether or not every pseudo-circle is a continuous image of the pseudo-arc, and it is not known whether or not every solenoid is a continuous image of a pseudo-circle. Solutions to each of these problems will be included in a subsequent paper to be presented by this author.

Added in proof. Professor F. Burton Jones has mentioned to this author that James T. Rogers Jr, one of his Ph. D. students, has recently obtained some of the results of this paper by independent investigation.

\section{REFERENCES}

1. R. H. Bing, A homogeneous indecomposable plane continuum, Duke Math. J. 15 (1948), 729-742.

2. - Concerning hereditarily indecomposable continua, Pacific J. Math. 1 (1951), $43-51$.

3. The pseudo-arc, Summary of Lectures and Seminars, Summer Institute on Set Theoretic Topology, Madison, Wis., 1955, American Mathematical Society, Providence, R.I., 70-73.

4. ﹎.___. Embedding circle-like continua in the plane, Canadian J. Math. 14 (1962), 113-128. 
5. C. E. Burgess, Chainable continua and indecomposability, Pacific J. Math. 9, (1959), 653-659.

6. L. Fearnley, Characterizations of the continuous images of the pseudo-arc, Trans. Amer. Math. Soc. 111 (1964), 380-399.

7. - Topological operations on the class of continuous images of all snake-like continua, Proc. London Math. Soc. 15 (1965), 289-300.

8. B. Knaster, Un continua dont tout sous-continua est indecomposables, Fund. Math. 3 (1922), 247-286.

9. A. Lelek, On weakly chainable continua, Fund. Math. 51 (1963), 271-283.

10. J. Mioduszewski, Functional conception of snake-like continua, Fund. Math. 51 (1962), 179-189.

11. E. E. Moise, An indecomposable continuum which is homeomorphic to each of its nondegenerate subcontinua, Trans, Amer. Math. Soc. 63 (1948), 581-594.

12. G. T. Whyburn, Analytic topology, Amer. Math. Soc. Colloquium Publications, vol. 38; American Mathematical Society, Providence, R.I., 1942.

Received June 7, 1965. This research was supported by the National Science Foundation under Grants NSF-GP2244 and GP6308.

Brigham Young UNIVERSity

UNIVERSITY OF WISCONSION 



\section{PACIFIC JOURNAL OF MATHEMATICS}

\section{H. ROYDEN}

Stanford University

Stanford, California

\author{
J. P. JANS \\ University of Washington \\ Seattle, Washington 98105
}

\section{EDITORS}

\author{
J. DugundJI \\ Department of Mathematics \\ Rice University \\ Houston, Texas 77001 \\ Richard ARENS \\ University of California \\ Los Angeles, California 90024
}

\section{ASSOCIATE EDITORS}
E. F. BECKENBACH
B. H. NeumanN
F. WOLF
K. YOSIDA

\section{SUPPORTING INSTITUTIONS}

\author{
UNIVERSITY OF BRITISH COLUMBIA \\ CALIFORNIA INSTITUTE OF TECHNOLOGY \\ UNIVERSITY OF CALIFORNIA \\ MONTANA STATE UNIVERSITY \\ UNIVERSITY OF NEVADA \\ NEW MEXICO STATE UNIVERSITY \\ OREGON STATE UNIVERSITY \\ UNIVERSITY OF OREGON \\ OSAKA UNIVERSITY \\ UNIVERSITY OF SOUTHERN CALIFORNIA
}

\author{
STANFORD UNIVERSITY \\ UNIVERSITY OF TOKYO \\ UNIVERSITY OF UTAH \\ WASHINGTON STATE UNIVERSITY \\ UNIVERSITY OF WASHINGTON \\ AMERICAN MATHEMATICAL SOCIETY \\ CHEVRON RESEARCH CORPORATION \\ TRW SYSTEMS \\ NAVAL ORDNANCE TEST STATION
}

Mathematical papers intended for publication in the Pacific Journal of Mathematics should be typewritten (double spaced). The first paragraph or two must be capable of being used separately as a synopsis of the entire paper. It should not contain references to the bibliography. Manuscripts may be sent to any one of the four editors. All other communications to the editors should be addressed to the managing editor, Richard Arens at the University of California, Los Angeles, California 90024.

50 reprints per author of each article are furnished free of charge; additional copies may be obtained at cost in multiples of 50 .

The Pacific Journal of Mathematics is published monthly. Effective with Volume 16 the price per volume (3 numbers) is $\$ 8.00$; single issues, $\$ 3.00$. Special price for current issues to individual faculty members of supporting institutions and to individual members of the American Mathematical Society: $\$ 4.00$ per volume; single issues $\$ 1.50$. Back numbers are available.

Subscriptions, orders for back numbers, and changes of address should be sent to Pacific Journal of Mathematics, 103 Highland Boulevard, Berkeley 8, California.

Printed at Kokusai Bunken Insatsusha (International Academic Printing Co., Ltd.), 7-17, Fujimi 2-chome, Chiyoda-ku, Tokyo, Japan.

PUBLISHED BY PACIFIC JOURNAL OF MATHEMATICS, A NON-PROFIT CORPORATION

The Supporting Institutions listed above contribute to the cost of publication of this Journal, but they are not owners or publishers and have no responsibility for its content or policies. 


\section{Pacific Journal of Mathematics}

A. A. Aucoin, Diophantine systems ............................. 419

Charles Ballantine, Products of positive definite matrices. I ............... 427

David Wilmot Barnette, A necessary condition for d-polyhedrality ............ 435

James Clark Beidleman and Tae Kun Seo, Generalized Frattini subgroups of finite groups ......................................... 441

Carlos Jorge Do Rego Borges, A study of multivalued functions ............. 451

William Edwin Clark, Algebras of global dimension one with a finite ideal

lattice ...............................................

Richard Brian Darst, On a theorem of Nikodym with applications to weak convergence and von Neumann algebras .........................

George Wesley Day, Superatomic Boolean algebras .....................

Lawrence Fearnley, Characterization of the continuous images of all

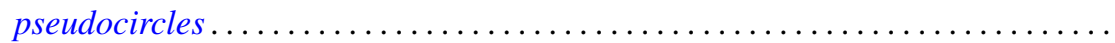

Neil Robert Gray, Unstable points in the hyperspace of connected subsets....... 515

Franklin Haimo, Polynomials in central endomorphisms .................. 521

John Sollion Hsia, Integral equivalence of vectors over local modular lattices . . . . 527

Jim Humphreys, Existence of Levi factors in certain algebraic groups .......... 543

E. Christopher Lance, Automorphisms of postliminal $C^{*}$-algebras ............ 547

Sibe Mardesic, Images of ordered compacta are locally peripherally metric . . . . 557

Albert W. Marshall, David William Walkup and Roger Jean-Baptiste Robert Wets,

Order-preserving functions: Applications to majorization and order

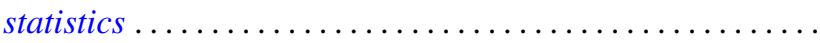

Wellington Ham Ow, An extremal length criterion for the parabolicity of

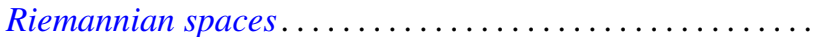

585

Wellington Ham Ow, Criteria for zero capacity of ideal boundary components of

Riemannian spaces...................................... 591

J. H. Reed, Inverse limits of indecomposable continua .................. 597

Joseph Gail Stampfli, Minimal range theorems for operators with thin spectra . . . 601

Roy Westwick, Transformations on tensor spaces..................... 613

Howard Henry Wicke, The regular open continuous images of complete metric

spaces ........................................... 621

Abraham Zaks, A note on semi-primary hereditary rings .............. 627

Thomas William Hungerford, Correction to: "A description of $\operatorname{Mult}_{i}\left(A^{1}, \cdots, A^{n}\right)$

by generators and relations" ............................. 629

Uppuluri V. Ramamohana Rao, Correction to: "On a stronger version of Wallis' formula".............................................. 629

Takesi Isiwata, Correction: "Mappings and spaces" ................... 630

Henry B. Mann, Josephine Mitchell and Lowell Schoenfeld, Correction to:

"Properties of differential forms in $n$ real variables" .... . .

James Calvert, Correction to: "An integral inequality with applications to the

Dirichlet problem"............................. 